\section{FRI0033 IMPORTANT ROLE OF CD11C+ DENDRITIC CELLS IN INFLAMMATORY ARTHRITIS}

A. Puchner $^{1}$, V. Saferding ${ }^{1}$, M. Bonelli ${ }^{1}$, H. Leiss ${ }^{1}$, R. Pfeifle ${ }^{2}$, G. Kroenke ${ }^{2}$, M. Koenders ${ }^{3}$, J. Smolen ${ }^{1}$, K. Redlich ${ }^{1}$, S. Blüml ${ }^{1} .{ }^{1}$ Medizinische Universität Wien, Wien, Austria; ${ }^{2}$ University Hospital Erlangen, Erlangen, Germany; ${ }^{3}$ Radboud University Nijmegen Medical Center, Nijmegen, Netherlands

Background: Dendritic cells (DCs) are important antigen presenting cells (APCs) and therefore they play an important role in bridging the innate and the adaptive immune response. DCs can be divided in different subsets with specific functions. As powerful APCs, DCs are thought to play an important role in the induction of autoimmune diseases such as rheumatoid arthritis. However, the active role of DCs in joint inflammation is not known yet.

Methods: We analyzed histological sections of $\mathrm{K} / \mathrm{BxN}$ serum transfer arthritis as well as hTNFtg arthritis for the presence of $\mathrm{CD} 11 \mathrm{c}^{+}$cells by immunohistochemistry. We also performed synovial biopsies and analyzed the cellular composition of the inflammatory infiltrate with respect to DCs. We used CD11c-diphteria toxin receptor (DTR) transgenic mice, which express the human diphtheria-toxin receptor under the $\mathrm{CD} 11 \mathrm{c}$ promoter, allowing for specific depletion of $\mathrm{CD} 11 \mathrm{c}^{+}$ cells by administration of diphtheria toxin (DT). K/BxN serum transfer arthritis was induced, and mice were given either DT or PBS or in wt and BARF3 deficient mice. In addition CD11c DTR mice were crossed into hTNFtg animals and also received either DT or PBS. The severity of arthritis was determined clinically and histologically.

Results: We show that $\mathrm{Cd}_{11 \mathrm{c}^{+}}$cells are present in significant numbers in the synovia of $\mathrm{K} / \mathrm{BxN}$ and TNF driven arthritis. Both $\mathrm{CD} 8^{+} \mathrm{CD} 11 \mathrm{c}^{+}$and $\mathrm{CD} 11 \mathrm{~b}^{+} \mathrm{CD} 11 \mathrm{c}^{+}$, can be found in synovial tissue. Upon depletion of $\mathrm{CD} 11 \mathrm{c}+$ cells clinical signs of K/BxN serum transfer arthritis were significantly reduced. Histological analysis found reduced synovial inflammation after the depletion of

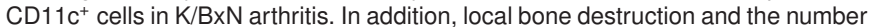
of osteoclasts was also significantly reduced. Analysis of $\mathrm{K} / \mathrm{BxN}$ arthritis in wt mice and BATF3 ${ }^{--}$mice, which lack a subset of DCs, namely CD8 ${ }^{+} \mathrm{CD} 11^{+} \mathrm{DCs}$, revealed no difference in arthritis severity between the two groups. In addition to $\mathrm{K} / \mathrm{BxN}$ arthritis, we found that also in TNF-driven arthritis depletion of $\mathrm{CD} 11 \mathrm{c}^{+}$ cells led to a striking reduction of synovial inflammation and a complete depletion of osteoclasts.

Conclusions: These data show that in addition to initiating an adaptive immune response, $\mathrm{CD} 11 \mathrm{c}^{+}$dendritic cells, are also involved in innate effector mechanisms of inflammatory arthritis. Especially $\mathrm{CD} 11 \mathrm{~b}^{+} \mathrm{CD} 11 \mathrm{c}^{+}$and monocyte derived inflammatory seem to play a role in inflammatory arthritis, suggesting that they could be an important therapeutic target for patients suffering from inflammatory arthritis.

Disclosure of Interest: None declared

DOI: 10.1136/annrheumdis-2017-eular.6625

\section{FRI0034 ADALIMUMAB REDUCES CXCR4 EXPRESSION DURING INFLAMMATORY ARTHRITIS AND IN FIBROBLAST-LIKE SYNOVIOCYTES AND OSTEOCLASTS UNDER CHRONIC TNF EXPOSURE}

B.P. Harvey ${ }^{1}$, L. Li ${ }^{2}$, M. Konrad ${ }^{2}$, H. Knight ${ }^{3}$, S. Westmoreland ${ }^{3}$, M. Ruzek ${ }^{2}$, Z. Kaymakcalan ${ }^{1} .{ }^{1}$ Biologics; ${ }^{2}$ Translational Research; ${ }^{3}$ Immunology Pharmacology, Abbvie Bioresearch Center, Worcester, United States

Background: The CXCL12/CXCR4 chemokine axis has been implicated in the pathogenesis of RA. The expression of this chemokine and receptor has been shown to be increased in RA synovium, and moreover, CXCR4 levels in synovium have been correlated with joint destruction in RA patients.

Objectives: Given that high levels of CXCR4 are associated with RA pathogenesis, we sought to determine whether CXCR4 levels are altered by adalimumab (ADA) both in vivo in RA patients and in the human TNF transgenic mouse model (huTNF Tg197) of arthritis, as well as in vitro with RA fibroblast-like synoviocytes (RA-FLS) and human osteoclast precursors (OCP) following TNF exposure. In addition, we investigated the role of CXCR4 in human osteoclastogenesis (OCgenesis) under chronic TNF exposure.

Methods: Public DNA microarray data of synovial tissue from ADA treated RA patients was analyzed for changes in the expression level of various chemokines and their cognate receptors. PBMC populations from ADA treated RA patients taken at baseline, wks. 4 \& 12 were assessed by CyTOF for CXCR4. IHC staining was performed on formalin paw sections from wk. 13 placebo control and ADA treated (1 mg/kg i.p.) huTNF Tg197 mice to evaluate CXCR4 expression in various pannus-associated cells. To assess the role of TNF and concomitant ADA treatment on human cultures in vitro, CXCR4 RNA expression was evaluated in RA-FLS treated with conditioned media from PBMCs +/-ADA for 6 hrs., and CXCR4 protein on OCP by flow cytometry in response to $72 \mathrm{hr}$. M-CSF+RANKL+/TNF+/-ADA. To demonstrate the role of CXCR4 in OCgenesis, OCP were cultured for $6 \mathrm{~d}$. in M-CSF+RANKL+/-TNF following 30 min. pretreatment with CXCR4 neutralizing antibody. OC maturation and activity were assessed by measuring TRAcP $5 b$ activity and CTX-I release, respectively.

Results: Preliminary microarray data analysis of RA synovial tissue demonstrated that active RA patients over-expressed CXCR4 while CXCR4 was significantly normalized (two-fold reduction) in the responders to ADA therapy. In addition, CyTOF analysis of PBMC from ADA treated RA patients indicated a significant 2-fold reduction from baseline in CXCR4 expression on B cells with a modest trend of reduction on CD4+ T cells by wk. 4. In huTNF Tg197 mice, CXCR4 expression in the inflamed pannus (most notably in FLS, lymphocytes and OC) was also decreased by ADA therapy. In vitro human cultures of similar cell types including RA-FLS and OCP were subjected to conditioned media or TNF, respectively, and found to express higher levels of CXCR4 that was reduced with concomitant ADA treatment. Finally, antibody-mediated blockade of CXCR4 on human OCP decreased both TNF-enhanced OC maturation and activity.

Conclusions: Our findings demonstrate that ADA therapy reduces CXCR4 expression in vivo both in RA patient PBMCs and in the pannus of huTNF Tg mice with inflammatory arthritis especially in lymphocytes, FLS and OC. Similar results were also observed with our in vitro human cultures of equivalent cell types. More importantly, we've shown that inhibition of CXCR4 can reduce TNF-enhanced OCgenesis in vitro, suggesting that CXCR4 may be a contributing factor to TNF-mediated osteolysis in RA.

Acknowledgements: Dr. J. Salfeld and J. Min

Disclosure of Interest: B. Harvey Shareholder of: AbbVie, Inc., Employee of: AbbVie, Inc., L. Li Shareholder of: AbbVie, Inc., Employee of: AbbVie, Inc., M. Konrad Shareholder of: AbbVie, Inc., Employee of: AbbVie, Inc., H. Knight Shareholder of: AbbVie, Inc., Employee of: AbbVie, Inc., S. Westmoreland Shareholder of: AbbVie, Inc., Employee of: AbbVie, Inc., M. Ruzek Shareholder of: AbbVie, Inc., Employee of: AbbVie, Inc., Z. Kaymakcalan Shareholder of: AbbVie, Inc., Employee of: AbbVie, Inc.

DOI: 10.1136/annrheumdis-2017-eular.2982

\section{FRI0035 SPECIFIC MONOCYTE SUBSETS IN PATIENTS WITH RHEUMATOID ARTHRITIS ARE ASSOCIATED WITH THE PROGRESSION OF THE DISEASE ALONG WITH THEIR AUTOIMMUNE AND PRO-ATHEROTHROMBOTIC PROFILE}

N. Barbarroja ${ }^{1}$, P. Ruiz-Limon ${ }^{1}$, C. Pérez-Sánchez ${ }^{1}$, Y. Jimenez-Gomez ${ }^{1}$, M.C. Abalos ${ }^{1}$, P. Segui ${ }^{2}$, R. Ortega ${ }^{1}$, E. Collantes ${ }^{1}$, A. Escudero ${ }^{1}$, M. Alarcon-Riquelme ${ }^{3}$, L. Lelann ${ }^{4}$, C. Jamin ${ }^{4}$, J.O. Pers ${ }^{4}$, C. Lopez-Pedrera ${ }^{1}$ on behalf of PRECISESADS Clinical Consortium. ${ }^{1}$ GC-5/Rheumatology;

${ }^{2}$ Radiology, IMIBIC/Reina Sofia Hospital/University of Cordoba, Cordoba; ${ }^{3}$ Genetic, Centro de Genomica e Investigación Oncológica (GENYO), Granada, Spain; ${ }^{4}$ Université de Brest, Brest, France

Background: Circulating monocytes play a key role in the pathogenesis of cardiovascular disease (CVD). Three monocyte subsets have been described based on their CD14 and CD16 expression profiles, with different actions in vascular pathology. The distribution of these monocyte subsets and their inflammatory profile associated with CVD in rheumatoid arthritis (RA) remain unknown.

Objectives: This study, developed within the Innovative Medicines Initiative Joint Undertaking (IMI JU) project PRECISESADS framework, aimed at: 1) determine which of the two CD16+ monocyte subpopulations is expanded in RA patients and to investigate their possible role in disease pathogenesis. 2) Functionally characterize the CD16+ monocyte subsets in RA patients and analyze their role in the pro-atherothrombotic profile associated to RA.

Methods: The frequencies of monocyte subpopulations in the peripheral blood of 50 healthy donors and 50 RA patients included in the PRECISESADS study were determined by flow cytometry. A second cohort of 30 RA patients was included, of which CD16+ monocyte subpopulation was isolated using immuno-magnetic selection. Proinflammatory circulating cytokines, as well as peroxide levels and cellular activation markers were analyzed. The expression of 84 genes related to atherosclerosis was analyzed by PCR-array. Endothelial function was measured through post occlusive hyperaemia using Laser-Doppler. Carotid intima media thickness (CIMT) was evaluated as atherosclerosis marker.

Results: CD14highCD16+ monocyte subset was expanded in RA patients belonging to the PRECISESADS study. Their frequency correlated with the evolution of the disease as well as with the positivity for RF and anti-CCP autoantibodies. Correlation studies further indicated a link between the increased frequency of these monocytes and increased levels of circulating cytokines such as IL-17F and IL-10.

CD14highCD16+ monocytes were also significantly extended in RA patients of the second cohort. Those patients had impaired endothelial function, with a reduced perfusion value after ischemia. Increased CD14highCD16+ monocyte proportions were associated with both endothelial dysfunction and the presence of a pathologic CIMT. CD16+ monocyte subsets showed increased protein and gene expression of proinflammatory cytokines, markers of atherosclerosis and peroxide levels.

Conclusions: RA patients exhibit an increased number of CD14highCD16+ monocytes, which display a specific atherogenic and inflammatory pattern directly associated to the autoimmune profile, the progression of the disease and the altered microvascular function. That data suggest that CD16+ subpopulation might play a key role in the CVD pathogenesis associated with RA.

Acknowledgements: Supported by the Innovative Medicines Initiative Joint Undertaking under grant agreement $n^{\circ} 115565$, resources of which are composed of financial contribution from the European Union's Seventh Framework Programme (FP7/2007-2013) and EFPIA companies' in kind contribution, the Instituto de Salud Carlos III (FIS PI15/1333; RIER Rd16/0012/0015) and the JA (CTS-7940). Disclosure of Interest: None declared

DOI: 10.1136/annrheumdis-2017-eular.4918 